\title{
Robust Control for Nonlinear Discrete-Time Systems with Quantitative Input to State Stability Requirement *
}

\author{
Shoudong Huang* Steven Weidong Su** \\ * ARC Centre of Excellence for Autonomous Systems (CAS), Faculty \\ of Engineering, University of Technology, Sydney, PO Box 123, \\ Broadway, NSW 2007, Australia (Tel: 61-2-95142964; e-mail: \\ sdhuang@eng.uts.edu.au). \\ ** Faculty of Engineering, University of Technology, Sydney, PO Box \\ 123, Broadway, NSW 2007, Australia (Tel: 61-2-95147603; e-mail: \\ Steven.Su@eng.uts.edu.au).
}

\begin{abstract}
In this paper, we consider state feedback robust control problems for discretetime nonlinear systems subject to disturbances. The objective of the control is to minimize a performance function while guaranteeing a prescribed quantitative input to state stability (ISS) property for the closed-loop systems. By introducing the concept of ISS control invariant set, a sufficient condition for the problem to be feasible is given. Built on the sufficient condition, a computationally efficient control design algorithm based on one-step min-max optimization is developed. An example is given to illustrate the proposed strategy.
\end{abstract}

Keywords: Input to state stability (ISS), nonlinear robust control, dynamic programming, controller synthesis, min-max optimization.

\section{INTRODUCTION}

The input to state stability (ISS) property is a robust stability property for nonlinear systems subject to disturbances. It was first proposed in Sontag (1989). Since then, the analysis and synthesis of ISS property has received a lot of attention with a range of applications reported in the literature. For example, a systematic analysis of the ISS property has been conducted in Sontag and Wang $(1995,1996)$, where its many different characterizations have been described. The ISS small-gain theorems and discrete-time ISS property were presented in Jiang et al. (1994) and Jiang and Wang (2001). The control system design to achieve closed-loop ISS is also called input-tostate stabilization. Most of the techniques for input-tostate stabilization are centered around the so called ISS control Lyapunov functions, see for example Krstic and $\mathrm{Li}$ (1998), Liberzon et al. (2002), and Malisoff et al. (2004).

Most of the work on analysis and synthesis of ISS had been focused on the qualitative results. Such as looking for qualitatively equivalent characterizations (Sontag and Wang (1996)) or designing system such that the closed-loop is ISS with some ISS gain and transient bound (e.g. Liberzon et al. (2002), Goularta et al. (2006), Kim et al. (2006), Lazar et al. (2008)). However, quantitative ISS results with explicitly prescribed ISS gain and transient bound requirement is more preferable in control system design. In our recent work Huang et al. (2005a), we considered the ISS synthesis problem when the ISS gains and bounds

\footnotetext{
* This work is supported by the ARC Centre of Excellence programme, funded by the Australian Research Council (ARC) and the New South Wales State Government.
}

on transients are given. We present a new technique for the synthesis of ISS that is based on a recently obtained result concerning $l^{\infty}$-bounded (LIB) robustness for nonlinear systems (Huang and James (2003)). By introducing two new state variables, the ISS synthesis problem is transformed into an equivalent uniform LIB dissipation synthesis problem and dynamic programming techniques are used to obtain necessary and sufficient conditions for the existence of state feedback and measurement feedback controller that result in the closed loop system to be ISS. Though the solutions in these work are optimal, the controller is dynamic and the computation cost is extremely high especially for higher order systems.

The focus of this paper is computationally efficient design algorithms to achieve prescribed quantitative ISS property. Since ISS is only a stability property, the control problem considered in this paper is to achieve a prescribed quantitative closed-loop ISS property while optimizing another performance index (either integral-type performance or hard bound type performance). The concept of ISS control invariant set is proposed and it is shown that the robust control problem is feasible as long as the initial state is within an ISS control invariant set. Furthermore, controller synthesis methods based on dynamic programming and one step min-max optimization are provided.

The paper is organized as follows. The ISS property is reviewed in Section 2. The state feedback control problems considered in this paper are stated in Section 3. In Section 4, the definition of ISS control invariant set is given and a sufficient condition for the state feedback control problems to be solvable is proved. Section 5 formulates the simplified 
problems and proposed the solutions using dynamic programming and one-step min-max optimization. In Section 6 , an example is given to illustrate the proposed min-max optimization strategy. Section 7 concludes the paper.

\section{THE ISS PROPERTY}

Sets of real numbers and nonnegative integers are denoted respectively as $\mathbf{R}$ and $\mathbf{Z}_{+}$. Recall that a function $\gamma$ : $[0, \infty) \rightarrow[0, \infty)$ is of class $\mathcal{K}$ if it is continuous, strictly increasing and $\gamma(0)=0$. A function $\beta:[0, \infty) \times[0, \infty) \rightarrow$ $[0, \infty)$ is said to be a function of class $\mathcal{K} \mathcal{L}$ if for each fixed $t \geq 0, \beta(\cdot, t)$ is of class $\mathcal{K}$ and for each fixed $s \geq 0, \beta(s, \cdot)$ decreases to zero.

Consider nonlinear discrete-time system

$$
x_{k+1}=f\left(x_{k}, w_{k}\right), k \geq 0 .
$$

Here $x_{k} \in \mathbf{R}^{n}, w_{k} \in \mathbf{W} \subseteq \mathbf{R}^{s}$ are the state and disturbance input, respectively. The function $f: \mathbf{R}^{n} \times$ $\mathbf{W} \rightarrow \mathbf{R}^{n}$ is well defined everywhere with $f(0,0)=0$. We exploit the following notation:

$$
\begin{aligned}
w_{0, k-1} & =\left\{w_{0}, \cdots, w_{k-1}\right\}, \forall k \geq 0, \\
\mathcal{W}_{0, k-1} & =\left\{w_{0, k-1}: w_{i} \in \mathbf{W}, 0 \leq i \leq k-1\right\}, \\
w_{0, \infty} & =\left\{w_{0}, \cdots, w_{k-1}, \cdots\right\}, \\
\mathcal{W}_{0, \infty} & =\left\{w_{0, \infty}: w_{i} \in \mathbf{W}\right\} .
\end{aligned}
$$

ISS Property: (Jiang and Wang (2001)) Nonlinear discrete-time system (1) is called input to state stable (ISS) if there exist $\beta \in \mathcal{K} \mathcal{L}$ and $\gamma \in \mathcal{K}$ such that the state trajectory satisfies

$$
\begin{aligned}
& \left|x_{k}\right| \leq \beta\left(\left|x_{0}\right|, k\right)+\gamma\left(\left\|w_{0, \infty}\right\|_{\infty}\right), \\
& \forall x_{0} \in \mathbf{R}^{n}, \forall w_{0, \infty} \in \mathcal{W}_{0, \infty}, \forall k \in \mathbf{Z}_{+}
\end{aligned}
$$

where

$$
\left\|w_{0, \infty}\right\|_{\infty}=\sup _{i \geq 0}\left|w_{i}\right|
$$

and $|\cdot|$ denotes a norm (e.g. 2-norm) of a vector.

By causality, the required inequality is equivalent to

$$
\begin{aligned}
& \left|x_{k}\right| \leq \beta\left(\left|x_{0}\right|, k\right)+\gamma\left(\left\|w_{0, k-1}\right\|_{\infty}\right), \\
& \forall x_{0} \in \mathbf{R}^{n}, \forall w_{0, k-1} \in \mathcal{W}_{0, k-1}, \forall k \in \mathbf{Z}_{+}
\end{aligned}
$$

where

$$
\left\|w_{0, k-1}\right\|_{\infty}=\max _{0 \leq i \leq k-1}\left|w_{i}\right| .
$$

Remark 2.1. A typical $\mathcal{K} \mathcal{L}$ function used in the ISS property is in the form

$$
\beta\left(\left|x_{0}\right|, k\right)=\left|x_{0}\right| a^{k},
$$

where $0<a<1$. That is, it is exponentially decaying in time step $k$. In this paper, we assume that the $\mathcal{K} \mathcal{L}$ functions in the ISS requirement is with the form (7).

\section{THE STATE FEEDBACK CONTROL PROBLEMS}

Consider nonlinear discrete-time control system

$$
\begin{aligned}
x_{k+1} & =f\left(x_{k}, u_{k}, w_{k}\right), k \geq 0 . \\
z_{k} & =g\left(x_{k}, u_{k}, w_{k}\right), k \geq 0 .
\end{aligned}
$$

Here $x_{k} \in \mathbf{R}^{n}, u_{k} \in \mathbf{U} \subseteq \mathbf{R}^{m}, w_{k} \in \mathbf{W} \subseteq \mathbf{R}^{s}, z_{k} \in$ $\mathbf{R}$ are the state, control input, disturbance input, and performance output, respectively. The functions $f: \mathbf{R}^{n} \times$ $\mathbf{U} \times \mathbf{W} \rightarrow \mathbf{R}^{n}$ and $g: \mathbf{R}^{n} \times \mathbf{U} \times \mathbf{W} \rightarrow \mathbf{R}$ are well defined everywhere.

The state feedback robust control problems considered in this paper are stated as follows.

Robust Control Problem I (using integral type performance): Given $\beta \in \mathcal{K} \mathcal{L}$ in the form of (7), $\gamma \in$ $\mathcal{K}$, and $x_{0} \in \mathbf{R}^{n}$, find, if possible, a control sequence $u_{0}, u_{1}, u_{2}, \cdots$ with $u_{k} \in \mathbf{U}(0 \leq k<\infty)$, such that the state trajectory of the resulting control system satisfies the ISS inequality

$$
\begin{aligned}
& \left|x_{k}\right| \leq \beta\left(\left|x_{0}\right|, k\right)+\gamma\left(\left\|w_{0, k-1}\right\|_{\infty}\right), \\
& \forall w_{0, k-1} \in \mathcal{W}_{0, k-1}, \forall k \in \mathbf{Z}_{+},
\end{aligned}
$$

and the integral-type performance

$$
\begin{aligned}
J_{\text {sum }} & =\sup _{w_{0, \infty} \in \mathcal{W}_{0, \infty}} \sum_{k=0}^{\infty} z_{k} \\
& =\sup _{w_{0, \infty} \in \mathcal{W}_{0, \infty}} \sum_{k=0}^{\infty} g\left(x_{k}, u_{k}, w_{k}\right),
\end{aligned}
$$

is minimized.

Robust Control Problem II (using hard bound type performance): Given $\beta \in \mathcal{K} \mathcal{L}$ as in (7), $\gamma \in \mathcal{K}$, and $x_{0} \in \mathbf{R}^{n}$, find, if possible, a control sequence $u_{0}, u_{1}, u_{2}, \cdots$ with $u_{k} \in \mathbf{U}(0 \leq k<\infty)$, such that the state trajectory of the control system satisfies the ISS inequality (9) and the hard bound type performance

$$
\begin{aligned}
J_{\text {max }} & =\sup _{w_{0, \infty} \in \mathcal{W}_{0, \infty}} \sup _{0 \leq k<\infty} z_{k} \\
& =\sup _{w_{0, \infty} \in \mathcal{W}_{0, \infty}} \sup _{0 \leq k<\infty} g\left(x_{k}, u_{k}, w_{k}\right),
\end{aligned}
$$

is minimized.

Remark 3.1. For control systems design, stability and performance are two key requirements. For nonlinear system with disturbances, a prescribed ISS property is a good stability requirement. For the performance, in some applications we may prefer integral type (e.g. minimize the total energy) and in some applications we may prefer hard bound type (e.g. minimize absolute tracking errors). Since stability is more critical, we treat the ISS inequality (robust stability) as a constraint.

Remark 3.2. The property described by inequality (9) is not a global ISS property since it is only required to hold for a given initial state $x_{0}$ and disturbances sequence $w_{0, \infty}$ within the set $\mathcal{W}_{0, \infty}$. Though inequality (9) is a weaker property than global ISS defined in Jiang and Wang (2001), the semi-global ISS defined in Nešić and Laila (2002), and the regional ISS defined in Magni et al. (2006), we believe that it is a reasonable requirement for state feedback nonlinear systems design since the system state is assumed to be available.

\section{ISS CONTROL INVARIANT SET}

The first important question to ask is when the Robust Control Problems I and II are feasible. That is, there exist at least one control sequence such that the ISS inequality (9) holds. 
The ISS requirement (9) can be regarded as an infinite number of state constraints for $k=1,2, \cdots$. Now we prove a simple sufficient condition for the ISS requirement to be satisfied.

Definition 4.1. Given $\mathbf{U} \subseteq \mathbf{R}^{m}, \mathbf{W} \subseteq \mathbf{R}^{s}, \beta \in \mathcal{K} \mathcal{L}$ as in (7), and $\gamma \in \mathcal{K}$, a set $S \subset \mathbf{R}^{n}$ is called an ISS controlled invariant set if $\forall x \in S$, there exists $u^{*}(x) \in \mathbf{U}$, such that

$$
\begin{gathered}
f\left(x, u^{*}(x), w\right) \in S \text { and } \\
\left|f\left(x, u^{*}(x), w\right)\right| \leq a|x|+(1-a) \gamma(|w|)
\end{gathered}
$$

for all $w \in \mathbf{W}$.

Lemma 4.2. Given $\mathbf{U} \subseteq \mathbf{R}^{m}, \mathbf{W} \subseteq \mathbf{R}^{s}, \beta \in \mathcal{K} \mathcal{L}$ as in (7), and $\gamma \in \mathcal{K}$, if $x_{0} \in S$ where $S$ is an ISS controlled invariant set defined in Definition 4.1, then the Optimal Control problems I and II are feasible. That is, there exists control sequence $u_{0}, u_{1}, u_{2}, \cdots$ such that the ISS requirement (9) is satisfied.

Proof: Suppose an arbitrary $x_{0} \in S$ is given.

At time $k=0$, since $x_{0} \in S$, we can choose $u_{0}=u^{*}\left(x_{0}\right)$, suppose the disturbance is $w_{0} \in \mathbf{W}$, then we have

$$
\begin{aligned}
x_{1} & =f\left(x_{0}, u_{0}, w_{0}\right) \in S \\
\left|x_{1}\right| & =\left|f\left(x_{0}, u_{0}, w_{0}\right)\right| \leq a\left|x_{0}\right|+(1-a) \gamma\left(\left|w_{0}\right|\right) .
\end{aligned}
$$

At time $k=1$, choose $u_{1}=u^{*}\left(x_{1}\right)$, suppose the disturbance is $w_{1} \in \mathbf{W}$, then we have

$$
\begin{aligned}
x_{2} & =f\left(x_{1}, u_{1}, w_{1}\right) \in S \\
\left|x_{2}\right| & =\left|f\left(x_{1}, u_{1}, w_{1}\right)\right| \leq a\left|x_{1}\right|+(1-a) \gamma\left(\left|w_{1}\right|\right) .
\end{aligned}
$$

Combining (13) and (14), we have

$$
\begin{aligned}
\left|x_{2}\right| & \leq a\left|x_{1}\right|+(1-a) \gamma\left(\left|w_{1}\right|\right) \\
& \leq a\left(a\left|x_{0}\right|+(1-a) \gamma\left(\left|w_{0}\right|\right)\right)+(1-a) \gamma\left(\left|w_{1}\right|\right) \\
& =a^{2}\left|x_{0}\right|+a(1-a) \gamma\left(\left|w_{0}\right|\right)+(1-a) \gamma\left(\left|w_{1}\right|\right) \\
& \leq a^{2}\left|x_{0}\right|+a(1-a) \gamma\left(\left\|w_{0,1}\right\|_{\infty}\right) \\
& \quad+(1-a) \gamma\left(\left\|w_{0,1}\right\|_{\infty}\right) \\
& =a^{2}\left|x_{0}\right|+(1+a)(1-a) \gamma\left(\left\|w_{0,1}\right\|_{\infty}\right) .
\end{aligned}
$$

In general, we will have $x_{k} \in S$ and

$$
\begin{aligned}
\left|x_{k}\right| & \leq a\left|x_{k-1}\right|+(1-a) \gamma\left(\left|w_{k-1}\right|\right) \\
& \leq a^{k}\left|x_{0}\right|+\left(1+a+a^{2}+\cdots+a^{k-1}\right) \\
& \cdot(1-a) \gamma\left(\left\|w_{0, k-1}\right\|_{\infty}\right) \\
& =a^{k}\left|x_{0}\right|+\left(1-a^{k}\right) \gamma\left(\left\|w_{0, k-1}\right\|_{\infty}\right) \\
& \leq a^{k}\left|x_{0}\right|+\gamma\left(\left\|w_{0, k-1}\right\|_{\infty}\right)
\end{aligned}
$$

This is the ISS inequality (9) with $\beta\left(\left|x_{0}\right|, k\right)=a^{k}\left|x_{0}\right|$. The proof is completed.

\section{THE SIMPLIFIED CONTROL PROBLEM AND SOLUTIONS}

Suppose there exist an ISS control invariant set $S$. For any $x \in S$, define the set of admissible control $U(x)$ as

$$
U(x)=\{u \in \mathbf{U}: \text { for all } w \in \mathbf{W},(12) \text { holds. }\} .
$$

By Definition 4.1, the set $U(x)$ is non-empty for any $x \in S$.

Once the ISS control invariant set $S$ and the set of admissible control $U(x), \forall x \in S$ are obtained, the robust control problems can be simplified and solved.

\subsection{The simplified problems}

Now the Robust Control Problems I and II are simplified as follows.

Simplified Problem I (using integral type performance): minimize

$$
J_{\text {sum }}=\sup _{w_{0, \infty} \in \mathcal{W}_{0, \infty}} \sum_{k=0}^{\infty} z_{k}
$$

subject to

$$
u_{k} \in U\left(x_{k}\right) .
$$

Simplified Problem II (using hard bound type performance): minimize

$$
J_{\max }=\sup _{w_{0, \infty} \in \mathcal{W}_{0, \infty}} \sup _{0 \leq k \leq \infty} z_{k}
$$

subject to (18).

\subsection{Solution using Dynamic Programming}

For the simplified problems, dynamic programming techniques (e.g. Huang and James (2003)) can be applied to solve the problems.

Simplified Problem I (using integral type performance) Define value function $V: S \rightarrow \mathbf{R}$ as

$$
\begin{aligned}
V(x)= & \inf _{u_{0, \infty}} \sup _{w_{0, \infty} \in \mathcal{W}_{0, \infty}}\left\{\sum_{k=0}^{\infty} g\left(x_{k}, u_{k}, w_{k}\right)\right. \\
& \left.: x_{k+1}=f\left(x_{k}, u_{k}, w_{k}\right), u_{k} \in U\left(x_{k}\right), x_{0}=x\right\} .
\end{aligned}
$$

The dynamic programming equation is

$$
V(x)=\inf _{u \in U(x)} \sup _{w \in \mathbf{W}}\{g(x, u, w)+V(f(x, u, w))\} .
$$

Simplified Problem II (using hard bound type performance) Define value function $V: S \rightarrow \mathbf{R}$ as

$$
\begin{aligned}
V(x)= & \inf _{u_{0, \infty}} \sup _{w_{0, \infty} \in \mathcal{W}_{0, \infty}}\left\{\sup _{\substack{0 \leq \infty \\
0 \leq k}} g\left(x_{k}, u_{k}, w_{k}\right)\right. \\
& \left.: x_{k+1}=f\left(x_{k}, u_{k}, w_{k}\right), u_{k} \in U\left(x_{k}\right), x_{0}=x\right\}
\end{aligned}
$$

The dynamic programming equation is

$$
V(x)=\inf _{u \in U(x)} \sup _{w \in \mathbf{W}} \max \{g(x, u, w), V(f(x, u, w)\} .
$$

Although dynamic programming approach gives the formula for optimal solution of the simplified problems, to solve the dynamic programming equation is not easy. Analytical solution to dynamic programming equation is usually impossible to derive. To obtain a numerical solution of the dynamic programming equation, a discretization over the set $S$ is necessary and the convergence depends on the nonlinear functions $f$ and $g$.

\subsection{Solution using one step min-max optimization}

Lemma 4.2 shows that the Optimal Control Problems can be greatly simplified by treating the ISS requirement as one constraint (18). However, the control problems are still infinite time horizon optimal control problems which are difficult to solve. 
Here we proposed a simplified solution to the problem using one step min-max optimization.

At time $k$, the following min-max optimization problem

$$
\inf _{u_{k} \in U\left(x_{k}\right)} \sup _{w_{k} \in \mathbf{W}} g\left(x_{k}, u_{k}, w_{k}\right)
$$

is solved and the solution $u_{k}^{*}$ is performed. Since $u_{k}^{*} \in$ $U\left(x_{k}\right)$, the ISS inequality (9) holds. Though the optimality is sacrificed as compared with dynamic programming approach, the computation cost is significantly reduced.

In summary, the whole controller design process using the one step min-max optimization can be stated as:

\section{Control design algorithm:}

- Step 1, compute the ISS control invariant set $S$ and the admissible control set $U(x)$ for $x \in S$.

- Step 2, at each time $k$, solve the one step min-max optimization problem (23), apply the control $u_{k}^{*}$.

\section{EXAMPLE}

Consider one-dimensional discrete-time system with dynamics:

$$
x_{k+1}=f\left(x_{k}, u_{k}, w_{k}\right)=x_{k}+\frac{1}{3} x_{k}^{3}+u_{k}+\frac{1}{2} w_{k}
$$

where the state $x_{k} \in \mathbf{R}$, the control $u_{k} \in \mathbf{U}=[-1,1]$, the disturbance input $w_{k} \in \mathbf{W}=[-1,1]$. Obviously, the system is unstable when $u_{k}=0$ and $w_{k}=0$. The performance function is supposed to be

$$
\begin{aligned}
z_{k} & =g\left(x_{k}, u_{k}, w_{k}\right)=x_{k+1}^{2}+u_{k}^{2}-w_{k}^{2} \\
& =\left(x_{k}+\frac{1}{3} x_{k}^{3}+u_{k}+\frac{1}{2} w_{k}\right)^{2}+u_{k}^{2}-w_{k}^{2} .
\end{aligned}
$$

Suppose the required ISS inequality is (9) with

$$
\beta(s, k)=s \cdot 2^{-k}, \quad \gamma(\delta)=\delta .
$$

For this example, $S=[-1,1]$ is an ISS control invariant set and the ISS admissible control set is given by $U(x)$ as follows.

If $x \geq 0$,

$$
\begin{aligned}
U(x) & =\left[-\frac{3}{2} x-\frac{1}{3} x^{3},-\frac{1}{2} x-\frac{1}{3} x^{3}\right] \bigcap[-1,1] \\
& =\left[\max \left\{-\frac{3}{2} x-\frac{1}{3} x^{3},-1\right\},-\frac{1}{2} x-\frac{1}{3} x^{3}\right] .
\end{aligned}
$$

If $x<0$,

$$
\begin{aligned}
U(x) & =\left[-\frac{1}{2} x-\frac{1}{3} x^{3},-\frac{3}{2} x-\frac{1}{3} x^{3}\right] \bigcap[-1,1] \\
& =\left[-\frac{1}{2} x-\frac{1}{3} x^{3}, \min \left\{1,-\frac{3}{2} x-\frac{1}{3} x^{3}\right\}\right] .
\end{aligned}
$$

In fact, for any $x \in S$ and any $u \in U(x)$,

$$
|f(x, u, w)|=\left|u+x+\frac{1}{3} x^{3}+\frac{1}{2} w\right| \leq \frac{1}{2}|x|+\frac{1}{2}|w|,
$$

This is inequality (12) with $a=\frac{1}{2}$.
Now the one step min-max optimization problem is:

$$
\begin{aligned}
& \inf _{u \in U(x)} \sup _{w \in \mathbf{W}} g(x, u, w) \\
&= \inf _{u \in U(x)} \sup _{w \in[-1,1]}\left\{\left(x+\frac{1}{3} x^{3}+u+\frac{1}{2} w\right)^{2}+u^{2}-w^{2}\right\} \\
&= \inf _{u \in U(x)} \sup _{w \in[-1,1]}\left\{\left(x+\frac{1}{3} x^{3}+u\right)^{2}+u^{2}\right. \\
&\left.-\frac{3}{4} w^{2}+\left(x+\frac{1}{3} x^{3}+u\right) w\right\} \\
&= \inf _{u \in U(x)} \sup _{w \in[-1,1]}\left\{\left(x+\frac{1}{3} x^{3}+u\right)^{2}+u^{2}\right. \\
&\left.-\frac{3}{4}\left[w-\frac{2}{3}\left(x+\frac{1}{3} x^{3}+u\right)\right]^{2}+\frac{1}{3}\left(x+\frac{1}{3} x^{3}+u\right)^{2}\right\} \\
&= \inf _{u \in U(x)} \sup _{w \in[-1,1]}\left\{\frac{4}{3}\left(x+\frac{1}{3} x^{3}+u\right)^{2}+u^{2}\right. \\
&\left.\quad-\frac{3}{4}\left[w-\frac{2}{3}\left(x+\frac{1}{3} x^{3}+u\right)\right]^{2}\right\} .
\end{aligned}
$$

By (27) and (28), when $x \in S$ and $u \in U(x)$,

$$
-\frac{1}{2}|x| \leq x+\frac{1}{3} x^{3}+u \leq \frac{1}{2}|x|,
$$

thus

$$
-\frac{1}{3} \leq-\frac{1}{3}|x| \leq \frac{2}{3}\left(x+\frac{1}{3} x^{3}+u\right) \leq \frac{1}{3}|x| \leq \frac{1}{3} .
$$

Since

we have

$$
-1 \leq \frac{2}{3}\left(x+\frac{1}{3} x^{3}+u\right) \leq 1
$$

$$
w^{*}=\frac{2}{3}\left(x+\frac{1}{3} x^{3}+u\right)
$$

in (29) and the optimization problem is

$$
\begin{aligned}
& \inf _{u \in U(x)}\left\{\frac{4}{3}\left(x+\frac{1}{3} x^{3}+u\right)^{2}+u^{2}\right\} \\
= & \inf _{u \in U(x)}\left\{\frac{7}{3} u^{2}+\frac{8}{3}\left(x+\frac{1}{3} x^{3}\right) u+\frac{4}{3}\left(x+\frac{1}{3} x^{3}\right)^{2}\right\} .
\end{aligned}
$$

The optimization problem can be solved easily as

$$
\begin{aligned}
& \inf _{u \in U(x)} \sup _{w \in \mathbf{W}} g(x, u, w) \\
= & \inf _{u \in U(x)}\left\{\frac{7}{3} u^{2}+\frac{8}{3}\left(x+\frac{1}{3} x^{3}\right) u+\frac{4}{3}\left(x+\frac{1}{3} x^{3}\right)^{2}\right\} \\
= & \inf _{u \in U(x)}\left\{\frac{7}{3}\left[u^{2}+\frac{8}{7}\left(x+\frac{1}{3} x^{3}\right) u\right]+\frac{4}{3}\left(x+\frac{1}{3} x^{3}\right)^{2}\right\} \\
= & \inf _{u \in U(x)}\left\{\frac{7}{3}\left[u+\frac{4}{7}\left(x+\frac{1}{3} x^{3}\right)\right]^{2}-\frac{7}{3} \cdot \frac{16}{49}\left(x+\frac{1}{3} x^{3}\right)^{2}\right. \\
& \left.+\frac{4}{3}\left(x+\frac{1}{3} x^{3}\right)^{2}\right\} \\
= & \inf _{u \in U(x)}\left\{\frac{7}{3}\left[u+\frac{4}{7}\left(x+\frac{1}{3} x^{3}\right)\right]^{2}+\frac{4}{7}\left(x+\frac{1}{3} x^{3}\right)^{2}\right\} .
\end{aligned}
$$

(i) If $x \geq 0$ and $x \leq \frac{\sqrt{2}}{2}$, then

$$
\frac{3}{7}\left(x+\frac{1}{3} x^{3}\right) \leq \frac{1}{2} x,
$$

and

$$
-\frac{1}{2} x \leq \frac{3}{7}\left(x+\frac{1}{3} x^{3}\right) \leq \frac{1}{2} x,
$$

thus

$$
-\frac{3}{2} x-\frac{1}{3} x^{3} \leq-\frac{4}{7}\left(x+\frac{1}{3} x^{3}\right) \leq-\frac{1}{2} x-\frac{1}{3} x^{3},
$$


since $x \leq 1$, we also have

$$
-1 \leq-\frac{4}{7} \cdot \frac{4}{3} \leq-\frac{4}{7}\left(x+\frac{1}{3} x^{3}\right)
$$

and thus by (27),

$$
u^{*}=-\frac{4}{7}\left(x+\frac{1}{3} x^{3}\right) \in U(x)
$$

with

$$
\inf _{u \in U(x)} \sup _{w \in \mathbf{W}} g(f(x, u, w), u, w)=\frac{4}{7}\left(x+\frac{1}{3} x^{3}\right)^{2} .
$$

(ii) If $x \geq 0$ and $x>\frac{\sqrt{2}}{2}$, then

$$
\frac{3}{7}\left(x+\frac{1}{3} x^{3}\right)>\frac{1}{2} x,
$$

and

and thus

$$
-\frac{4}{7}\left(x+\frac{1}{3} x^{3}\right)>-\frac{1}{2} x-\frac{1}{3} x^{3}
$$

with

$$
u^{*}=-\frac{1}{2} x-\frac{1}{3} x^{3} \in U(x)
$$

$$
\begin{gathered}
\inf _{u \in U(x)} \sup _{w \in \mathbf{W}} g(f(x, u, w), u, w) \\
=\frac{7}{3}\left(\frac{1}{14} x-\frac{1}{7} x^{3}\right)^{2}+\frac{4}{7}\left(x+\frac{1}{3} x^{3}\right)^{2} .
\end{gathered}
$$

(iii) If $x \leq 0$ and $x \geq-\frac{\sqrt{2}}{2}$, then

$$
\frac{3}{7}\left(x+\frac{1}{3} x^{3}\right) \geq \frac{1}{2} x,
$$

then

$$
\frac{1}{2} x \leq \frac{3}{7}\left(x+\frac{1}{3} x^{3}\right) \leq-\frac{1}{2} x
$$

thus

$$
-\frac{1}{2} x-\frac{1}{3} x^{3} \leq-\frac{4}{7}\left(x+\frac{1}{3} x^{3}\right) \leq-\frac{3}{2} x-\frac{1}{3} x^{3},
$$

since $x \geq-1$ we also have

by (28),

$$
-\frac{4}{7}\left(x+\frac{1}{3} x^{3}\right) \leq \frac{4}{7} \cdot \frac{4}{3} \leq 1,
$$

with

$$
u^{*}=-\frac{4}{7}\left(x+\frac{1}{3} x^{3}\right) \in U(x)
$$

$$
\inf _{u \in U(x)} \sup _{w \in \mathbf{W}} g(f(x, u, w), u, w)=\frac{4}{7}\left(x+\frac{1}{3} x^{3}\right)^{2} .
$$

(iv) If $x \leq 0$ and $x<-\frac{\sqrt{2}}{2}$, then

$$
\frac{3}{7}\left(x+\frac{1}{3} x^{3}\right)<\frac{1}{2} x,
$$

and

$$
-\frac{4}{7}\left(x+\frac{1}{3} x^{3}\right)<-\frac{1}{2} x-\frac{1}{3} x^{3},
$$

thus

$$
u^{*}=-\frac{1}{2} x-\frac{1}{3} x^{3} \in U(x)
$$

with

$$
\begin{gathered}
\inf _{u \in U(x)} \sup _{w \in \mathbf{W}} g(f(x, u, w), u, w) \\
=\frac{7}{3}\left(\frac{1}{14} x-\frac{1}{7} x^{3}\right)^{2}+\frac{4}{7}\left(x+\frac{1}{3} x^{3}\right)^{2} .
\end{gathered}
$$

In summary, the control law obtained by the one step minmax optimization is

$$
u^{*}(x)=\left\{\begin{array}{l}
-\frac{4}{7}\left(x+\frac{1}{3} x^{3}\right), \text { if }-\frac{\sqrt{2}}{2} \leq x \leq \frac{\sqrt{2}}{2} \\
-\frac{1}{2} x-\frac{1}{3} x^{3}, \quad \text { if } x<-\frac{\sqrt{2}}{2} \text { or } x>\frac{\sqrt{2}}{2} .
\end{array}\right.
$$

The state feedback control law (36) is given in (a) of Figure 1. A simulation of the closed-loop system is illustrated in (b)-(d) of Figure 1, which demonstrates consistency with the ISS requirement.

\section{CONCLUSION}

The state feedback robust control design problems with quantitative input-to-state stability (ISS) requirement are studied. The concept of ISS control invariant set is proposed to derive sufficient conditions for the problems to be solvable. The one-step min-max optimization is applied for the robust controller design. The key difference between the problems considered in this paper and those considered in the ISS control design literature is that the ISS requirement here is quantitative. As such, the ISS control invariant set we defined depends on the particular quantitative ISS requirement.

Just like the importance of other invariant sets in control reviewed in Blanchini (1999), ISS control invariant set plays a key role in the system design to achieve ISS. However, the computation of the ISS control invariant set is not trivial for general nonlinear systems. We are currently working on numerical methods for computing the ISS control invariant set. We are also looking at the Model Predictive Control strategy to solve the problem such that the system performance may be improved. The corresponding measurement feedback robust control design problems are more challenging and is our future research work.

\section{REFERENCES}

F. Blanchini, Set invariance in control, Automatica, 35, 1747-1767, 1999.

P.J. Goularta, E.C. Kerriganb, and J.M. Maciejowski, Optimization over state feedback policies for robust control with constraints, Automatica, 42, 523-533, 2006.

S. Huang and M.R. James, $l^{\infty}$-bounded robustness for nonlinear systems: analysis and synthesis, IEEE Trans. on Automatic Control. 48(11), 1875-1891, 2003.

S. Huang, M.R. James, D. Nešić and P. Dower, A unified approach to controller design for achieving ISS and related properties, IEEE Trans. on Automatic Control. Vol.50, No.11, 1681-1697, 2005.

S. Huang, M.R. James, D. Nešić and P. Dower, Analysis of input to state stability for discrete time nonlinear systems via dynamic programming, Automatica. Vol.41, No.12, 2055-2065, 2005.

Z.P. Jiang and A.R. Teel and L.Praly, Small-gain theorem for ISS systems and applications, Mathematics of Control, Signals, and Systems, 7, 95-120, 1994.

Z.P. Jiang and Y. Wang, Input-to-state stability for discrete-time nonlinear systems, Automatica, 37, 857869, 2001. 


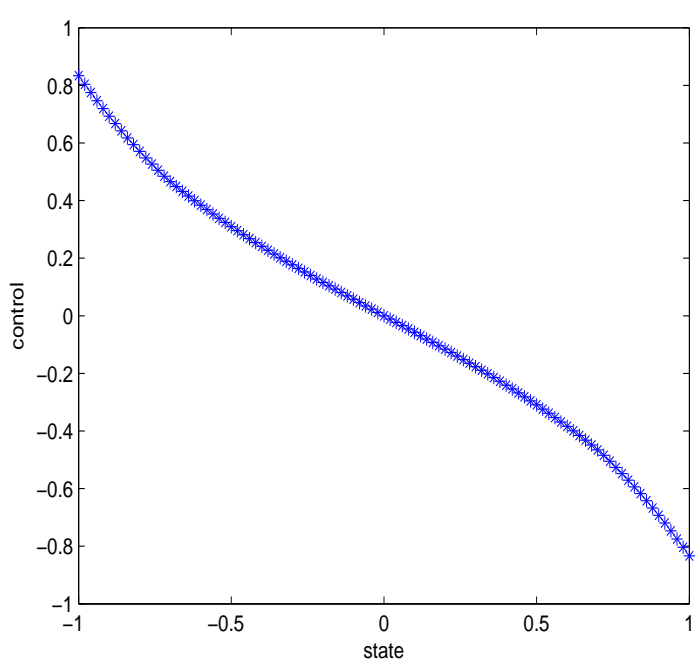

(a) state feedback controller $u^{*}(x)$

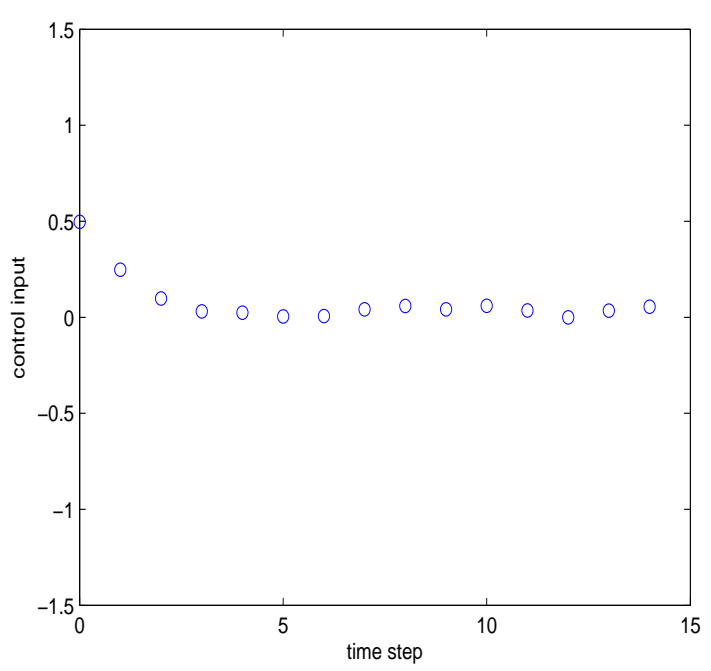

(c) the control input sequence

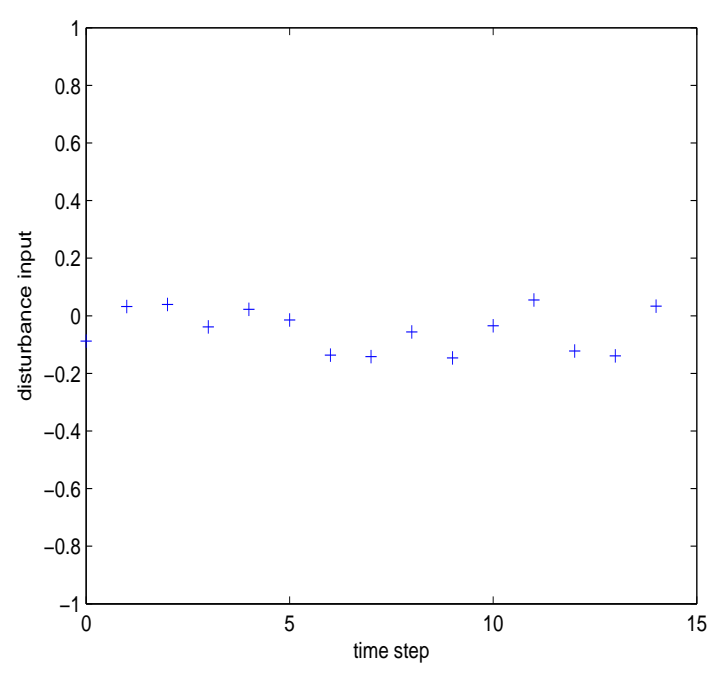

(b) the disturbance signal sequence

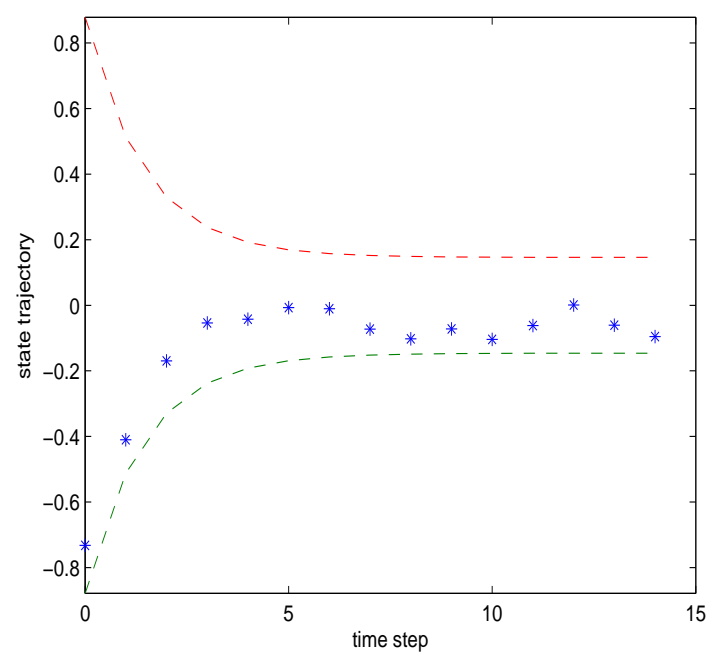

(d) the state trajectory and the required ISS bound

Fig. 1. state feedback controller and a trajectory of closed-loop system

J.S. Kim, T.W. Yoon, A. Jadbabaie, C. De Persis, Inputto-state stable finite horizon MPC for neutrally stable linear discrete-time systems with input constraints, Systems \& Control Letters, 55, 293-303, 2006.

M. Krstic and Z. Li, Inverse optimal design of input-tostate stabilizing nonlinear controllers, IEEE Trans. Aut. Control, 43, 1998, pp. 336-350.

M. Lazar, D. Munoz de la Pena, W.P.M.H. Heemels and T. Alamo, On input-to-state stability of min-max nonlinear model predictive control, Systems \& Control Letters, 57, 39-48, 2008.

D. Liberzon, E.D. Sontag and Y. Wang, Universal construction of feedback laws achieving ISS and integral-ISS disturbance attenuation, Systems \& Control Letters, 46, 111-127, 2002.

L. Magni, D.M. Raimondo, and R.D. Scattolini, Regional input-to-state stability for nonlinear model predictive control, IEEE Trans. Autom. Control, 51 (9): 1548-1553 SEP 2006.
M. Malisoff, L. Rifford, and E. Sontag, Global asymptotic controllability implies input to state stabilization, SIAM J. Control Optim. Vol. 42, No. 6, pp. 2221-2238, 2004.

D. Nešić and D.S. Laila, A note on input-to-state stabilization for nonlinear sampled-data systems, IEEE Trans. Autom. Control, vol. 47, no. 7, pp. 1153-1158, Jul. 2002.

E.D. Sontag, Smooth stabilization implies coprime factorization, IEEE Trans. Aut. Control, 34, 435-443, 1989.

E.D. Sontag and Y. Wang, On characterizations of input to state stability property, Systems \& Control Letters, 24, 351-359, 1995.

E.D. Sontag and Y. Wang, New characterizations of input to state stability property, IEEE Trans. Aut. Control, 41, 1283-1294, 1996. 